\title{
Optimización del método SCAR (Sequence Characterized Amplified Region) que favorece el aislamiento de loci polimórficos para estudios filogenéticos en taxa cercanamente relacionados
}

\author{
Optimization to the SCAR (Sequence Characterized Amplified Region) favors the isolation of \\ polymorphic loci for phylogenetic studies in closely related taxa
}

\author{
Dolores González \\ Red de Biodiversidad y Sistemática, Instituto de Ecología, A.C., Carretera Antigua a Coatepec 351, Congregación El Haya, 91070 Xalapa, \\ Veracruz, México. \\ Correspondencia: dolores.gonzalez@inecol.edu.mx
}

\begin{abstract}
Resumen. Las secuencias génicas han contribuido enormemente a los estudios filogenéticos en plantas. Sin embargo, una desventaja importante en las secuencias de varios loci es su baja resolución filogenética para resolver las relaciones de taxa cercanamente relacionados. En este trabajo se propone una modificación al método SCAR que consiste en: $a$ ) digerir fragmentos polimórficos generados con marcadores RAPD en lugar de clonarlos, $b$ ) secuenciar los fragmentos digeridos y c) alinear las secuencias para diseñar oligos específicos. Esta estrategia permitió comparar de manera más rápida y sencilla regiones variables útiles para los análisis filogenéticos en rangos taxonómicos menos inclusivos.
\end{abstract}

Palabras clave: filogenia molecular, marcadores moleculares, sistemática de plantas, enzimas de restricción, SCARDigerido, RAPD.

\begin{abstract}
DNA sequence data have made a tremendous contribution to plant phylogenetics. Nonetheless, a notable shortcoming of several loci has been the poor phylogenetic resolution of relationships among closely related species. In this study a modification to the SCAR method is proposed that consists of: a) digestion of polymorphic RAPD fragments instead of cloning them, $b$ ) sequencing of the digested fragments, and c) alignment of sequences for designing specific primers. This simple approach allowed us to find variable DNA loci suitable for phylogenetic analysis at lower taxonomic ranks.
\end{abstract}

Key words: molecular phylogeny, molecular markers, plant systematics, restriction enzymes, Digested-SCAR, RAPD.

Durante las pasadas 2 décadas, las secuencias de ADN desempeñaron un papel central en los estudios taxonómicos de plantas; han aportado una cantidad casi interminable de caracteres para estudios filogenéticos, y han permitido la comparación de grupos divergentes para los cuales sería difícil establecer homologías morfológicas. Aunque las secuencias se obtuvieron de los 3 genomas de plantas, muchas sólo han ayudado a resolver las relaciones filogenéticas en rangos taxonómicos inclusivos (Soltis y Soltis, 1998) y para muchos grupos se mantiene como un problema el resolver las relaciones filogenéticas entre taxa cercanamente relacionados (Crawford y Mort, 2004; Mort y Crawford, 2004).

Recibido: 23 enero 2009; aceptado: 10 julio 2009
Las regiones intergénicas del genoma se han recomendado para resolver las relaciones filogenéticas en rangos taxonómicos poco inclusivos porque sus funciones están menos restringidas y por lo tanto acumulan más variación, lo que potencialmente provee con más caracteres filogenéticos por loci secuenciado (Small et al., 1998; González y Vovides, 2002). En plantas, las regiones intergénicas del ADN del cloroplasto y núcleo son, por mucho, las secuencias favoritas para estudios filogenéticos a nivel de especies. Sin embargo, es frecuente que estas secuencias no sean suficientemente variables para proveer los niveles deseados de resolución y apoyo a los clados (Soltis y Soltis, 1998; Potter et al., 2000; Bailey et al., 2004). En estudios filogenéticos recientes, con las regiones intergénicas del trnL-F del ADN de cloroplasto y la región 
del ITS del ADN ribosómico nuclear, en los géneros Ceratozamia y Dioon (Zamiaceae, Cycadales) se observó muy poca variación entre las especies (González y Vovides, 2002; González et al., 2008). Los mismos resultados se encontraron en estudios desarrollados en nuestro laboratorio, utilizando las mismas regiones en especies de Tillansia (Bromeliaceae) y Beaucarnea (Agavaceae). La variación en nucleótidos resultó muy baja, contrastando de manera significativa con la gran variación morfológica que se observa entre las especies de estos géneros (Horres et al., 2000; Bogler y Francisco-Ortega, 2004; Barfuss, 2005; González et al., 2008).

Enun estudio reciente se utilizóla metodologíaSequence Characterized Amplified Region (SCAR) para identificar loci polimórficos en la reconstrucción filogenética en nivel específico (Paran y Michelmore, 1993; Bailey et al., 2004). Sin embargo, esta estrategia implica un paso laborioso que consiste en clonar los fragmentos polimórficos que se generan en un ensayo inicial con la metodología Random Amplified Polymorphic DNA (RAPD), antes de secuenciarlos. En los RAPD, los 2 extremos 5' de los amplicones que se generan contienen la misma secuencia del oligonucleótido (oligo) usado. Esta condición impide secuenciarlos en forma directa, ya que el ADN polimerasa utilizaría simultáneamente las 2 cadenas como templados, generando secuencias ilegibles. En consecuencia, en el método SCAR se clonan los amplicones con el objetivo de usar las secuencias del vector como oligos y así poder secuenciarlos (Paran y Michelmore, 1993). Por tal motivo, una estrategia donde no fuera necesario clonar estos segmentos resultaría más rápida y sencilla. En esta nota, se describe una optimización del método SCAR para detectar loci polimórficos para los análisis filogenéticos en especies cercanamente relacionadas, recientemente divergentes o en taxa infraespecíficos.

La optimización del método se desarrolló con el género Ceratozamia (Zamiaceae, Cycadales); primero consistió en explorar la variación genética en 5 especies con 6 oligos para los RAPD. Los marcadores RAPD revelan pequeñas diferencias genéticas, debido a que se explora una gran parte del genoma; son baratos, sencillos y ya existe una gran cantidad de oligos disponibles comercialmente. De los 6 probados, el OPB7 y el OPB10 (Operon Technologies) produjeron algunas bandas polimórficas durante la inspección de la variación genética. El intervalo en el tamaño de los amplicones osciló de 240 a 800 pares de bases (pb). Con la finalidad de generar las secuencias de estos fragmentos, los que presentaron una longitud entre 500 a 800 pb, en por lo menos 2 de las 5 especies, se cortaron del gel y se purificaron con el Wizard SV gel and PCR-clean up system (Promega, EUA). La purificación se recuperó en un volumen de $100 \mu$ l en una concentración de 600-800 ng.

En lugar de clonar los amplicones, como el método SCAR recomienda, el ADN purificado se dividió en 4 tubos para digerir con 4 enzimas de restricción de corte frecuente (Alu I, Rsa I, Bst OI y Taq I). La selección de estas enzimas fue arbitrario, sólo se consideró que su sitio de corte fuera de 4 nucleótidos para aumentar la probabilidad de que se encuentre un sitio de restricción en el fragmento. Por otro lado, cuando la concentración de algún amplicón es baja, se recomienda reamplificarlo antes de digerirlo.

El ADN digerido se sometió a una electroforesis horizontal en gel de agarosa al $1.2 \%$ por 2.5 horas a 60 volts. Después de la electroforesis de los productos digeridos, se observó que uno de los amplicones que se obtuvo con el oligo OPB7 en el ensayo inicial con RAPD tuvo un sitio de restricción de la enzima Bst OI, lo que separó los 2 extremos 5' que contenían el mismo sitio del oligo. Los segmentos resultantes se cortaron del gel y se purificaron con el método antes descrito. De la purificación, se tomaron alrededor de $20 \mathrm{ng}$ y se secuenciaron con el mismo oligo OPB7. Esta simple modificación redujo el trabajo de manera considerable y resultó en un método más sencillo y rápido para identificar loci polimórficos para análisis filogenéticos.

Las secuencias que se obtuvieron para ese fragmento (ahora llamado fragmento SCAR-Digerido) en por lo menos 2 especies de Ceratozamia se alinearon manualmente. Después de una inspección visual de los 2 extremos de las secuencias alineadas se seleccionaron 2 regiones con características apropiadas para generar oligos específicos con el fin de amplificar este fragmento en todas las especies de Ceratozamia. En este punto, la decisión de realizar sólo una reacción de secuencias para diseñar los oligos en ambas terminaciones 5' depende del tamaño de los fragmentos SCAR-Digeridos que se obtengan. Por ejemplo, si el amplicón seleccionado es de 800 pb y después de la digestión se generan 2 segmentos, uno de 700 y otro de 100 , podría decidirse sólo generar oligos para el fragmento de $700 \mathrm{pb}$, ya que con una sola reacción de secuenciación se podrían obtener los nucleótidos de este segmento en su totalidad y con ellos diseñar los oligos para ambos extremos (Fig. 1).

Los oligos recientemente diseñados para el fragmento SCAR-Digerido se usaron con éxito para amplificar un segmento de ADN en todas las especies de Ceratozamia. Este método simplificado permitió comparar de una forma más rápida loci variables para especies cercanamente relacionadas, como el caso de Ceratozamia. Aunque se encontró que la variación genética es baja en este linaje de plantas, el fragmento secuenciado mostró mayor variación (1.7\%) que la encontrada en regiones intergénicas del ADN de cloroplasto estudiadas con anterioridad para este grupo 


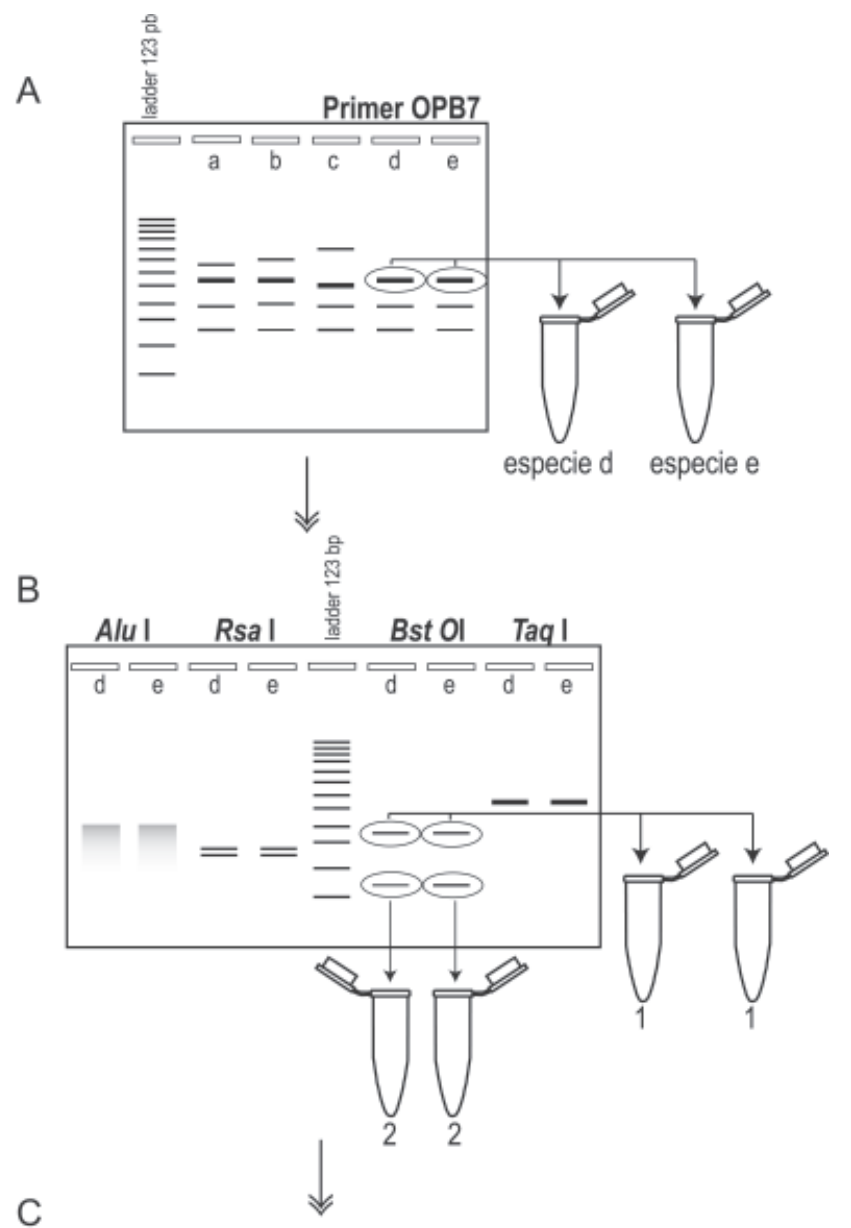

Alineamiento de las secuencias de los fragmentos 1 y 2

\section{Diseño de primers para los fragmentos 1 y 2}

Figura 1. Estrategia usada para detectar loci polimórficos en la reconstrucción filogenética delos taxa cercanamente relacionados, especies recientemente divergentes o taxa infraespecíficos. A). Purificación del fragmento de RAPD y digestión con enzimas de restricción de corte frecuente. B). Purificación de los fragmentos SCAR-digeridos $(1,2)$ y secuenciación con el primer RAPD (OPB7). C). Diseño de nuevos oligonucleótidos a partir de las secuencias alineadas de los fragmentos SCAR-Digeridos; a-e indican especies diferentes.

(0.2\%; González y Vovides, 2002). Este método presenta la desventaja de que las secuencias generadas están restringidas sólo a la reconstrucción filogenética en especies de Ceratozamia. Por ahora, no es posible compararlas con otros grupos de plantas pues se desconoce su procedencia. No obstante, este procedimiento eliminó la restricción de trabajar con regiones, que aunque conocidas, son poco variables. En la actualidad también se examina la utilidad de aplicar esta estrategia para encontrar variación genética dentro de poblaciones de la planta mexicana Beaucarnea recurvata Lem. (Agavaceae). La metodología que aquí se presenta es una opción viable para encontrar de una manera más eficiente loci variables para estudios filogenéticos en taxa cercanamente relacionados, recientemente divergentes o infraespecíficos. Esta estrategia también puede adoptarse para desarrollar marcadores moleculares especie-específicos con el propósito de identificación.

\section{Literatura citada}

Bailey, C. D., C. E. Hughes y S. A. Harris. 2004. Using RAPDs to identify DNA sequence loci for species level phylogeny reconstruction: an example from Leucaena (Fabaceae). Systematic Botany 29:4-14.

Barfuss, M., R. Samuel, W. Till y T. F. Stuessy. 2005. Phylogenetic relationships in subfamily Tillandsioideae (Bromeliaceae) based on DNA sequence data from seven plastid regions. American Journal of Botany 92:337-351.

Bogler, D. J. y J. Francisco-Ortega. 2004. Molecular systematic studies in cycads: evidence from $t r n \mathrm{~L}$ intron and ITS2 rDNA sequences. Botanical Review 70:260-273.

Crawford, D. J. y M. E. Mort. 2004. Single-locus molecular markers for inferring relationships at lower taxonomic levels: observations and comments. Taxon 53:631-635.

González, D. y A. P. Vovides. 2002. Low intralineage divergence in Ceratozamia (Zamiaceae) detected with nuclear ribosomal DNA ITS and chloroplast DNA trnL-F non-coding region. Systematic Botany 27:654-661.

González, D., A. P. Vovides y C. Bárcenas. 2008. Phylogenetic relationships of the Neotropical genus Dioon (Cycadales, Zamiaceae) based on nuclear and chloroplast DNA sequence data. Systematic Botany 33:229-236.

Horres, R., G. Zizka, G. Khal y K. Weising. 2000. Molecular phylogenetics of Bromeliaceae: evidence from trnL (UAA) intron sequences of the chloroplast genome. Plant Biology 2:306-315.

Mort, M. E. y D. J. Crawford. 2004. The continuing search: low-copy nuclear sequences for lower-level plant molecular phylogenetic studies. Taxon 53:257-261.

Paran, I. y R. W. Michelmore. 1993. Development of reliable PCR based markers linked to downy mildew resistance genes in lettuce. Theoretical and Applied Genetics 85:985-993.

Potter, D., J. J. Luby y R. E. Harrison. 2000. Phylogenetic relationships among species of Fragaria (Rosaceae) inferred from non-coding nuclear and chloroplast DNA sequences. Systematic Botany 25:337-348.

Small, R. L., J. A. Ryburn, R. C. Cronn, T. Seelanan y J. F. Wendel. 1998. The tortoise and the hare: choosing between noncoding plastome and nuclear $A d h$ sequences for phylogeny reconstruction in a recently diverged plant group. American Journal of Botany 85:1301-1315.

Soltis, D. E. y P. S. Soltis. 1998. Choosing an approach and an appropriate gene for phylogenetic analysis. In Molecular systematics of plants II, D. E. Soltis, P. S. Soltis y J. F. Doyle (eds.). Kluwer Academic, New York. p. 1-42. 